\section{Genetic analysis of a Siamese cat with Xanthinuria}

\section{Anabelle Ludi, Alexandra Kehl, Nicole Stieger, Ines Langbein-Detsch, Elisabeth Mueller}

Laboklin, Bad Kissingen, Germany

\section{OBJECTIVES}

Xanthinuria is reported as a hereditary disease in humans and dogs. In cats, Xanthinuria occurs very rarely, with a defect in xanthine-dehydrogenase (XDH) being discussed as cause. XDH is an enzyme that participates in purine metabolism catalyzing chemical degradation of xanthine to uric acid. Accumulation of xanthine can lead to health problems such as xanthine urinary stones. Aim of the study was to analyze the XDH gene of an affected cat to find variants as putative cause for xanthinuria.

\section{METHODS}

In one young Siamese cat with signs of stranguria and hematuria xanthine urinary stones were found. The cat was not treated with allopurinol, suggesting an inherited cause for the disease. Sanger sequencing of all exons and exonintron-boundaries of $X D H$ was performed. Using multialignment tools the base pair and amino acid sequences were compared with the current standard cat genome (Felis catus 9.0) and the one published by Tsuchida et al. (2000).

\section{RESULTS}

The references differed in 23 positions from each other leading to conservative amino acid replacements. Comparison of the amino acid sequence of the affected cat with the reference genome Felis catus 9.0 showed six conservative replacements and two non-conservative variants in the diseased Siamese cat (Leu249His and Arg385Trp).

\section{STATEMENT (CONCLUSIONS)}

Our experiments strengthen the hypothesis that xanthinuria in the affected Siamese cat is a genetic disorder It remains to be examined if the two found variants Leu249His and Arg385Trp have an impact in the enzymatic activity of XDH. Testing more affected and healthy Siamese cats is planned to strengthen the hypothesis.
The effect of nonabsorbent hydrophobic sand litter on urine protein-to-creatinine ratio (UPC) in cats

\section{Josh Kennils, Christina Maunder, Marta Costa}

The University of Bristol, Bristol, United Kingdom

\section{OBJECTIVES}

To investigate whether a commonly used non-absorbent litter affects UPC measurements in cats.

\section{METHODS}

Baseline UPCs of 40 urine samples obtained by cystocentesis from 39 cats were measured in duplicate. Up to $3 \mathrm{ml}$ of urine were placed in a Petri dish containing $4 \mathrm{~g}$ of non-absorbent hydrophobic sand litter and covered for 24-hours. Urine was recovered using a pipette and repeat UPCs measured in duplicate. Statistical analyses were performed using a commercially available software package. The difference between baseline and 24-hour UPCs was assessed using the Wilcoxon signed-rank test. Correlation was assessed using the Spearman rank test and agreement between measurements was assessed using Passing-Bablok regression and Bland-Altman analysis. Agreement for classification of proteinuria according to the International Renal Interest Society (IRIS) was assessed using Cohen's kappa.

\section{RESULTS}

UPCs ranged from 0.05 to 5.59 . There was a statistically significant decrease in UPC after exposure to nonabsorbent litter for 24-hours, $p<0.0005$. There was statistically significant, strong positive correlation between baseline UPCs and 24-hour UPCs, $r_{s}=0.988$. PassingBablok regression analysis revealed minimal constant and proportional error with an equation of $y=-0.0038+$ $0.9606 x$. Visual assessment of the Bland-Altman plot demonstrated excellent agreement between baseline UPCs and 24-hour UPCs. Cohen's kappa coefficient was $\kappa=0.915$, corresponding to almost perfect agreement

\section{STATEMENT (CONCLUSIONS)}

The agreement measures of Bland-Altman analysis and Cohen's kappa for IRIS proteinuria classification both suggest that the use of non-absorbent hydrophobic sand litter has minimal effect on UPC measurement in feline urine and that samples exposed to this litter substrate are acceptable for analysis. 